\title{
Accuracy of intraocular lens power calculation in paediatric cataract surgery
}

\author{
C Tromans, P M Haigh, S Biswas, I C Lloyd
}

\begin{abstract}
Aims-To determine the accuracy of intraocular lens (IOL) power calculation in a group of pseudophakic children.

Methods-A retrospective analysis of biometric and refractive data was performed on 52 eyes of 40 infants and children, who successfully underwent cataract extraction and IOL implantation. The following parameters were included: age at the time of surgery, keratometry, axial length, estimated refraction, and the power of IOL implanted. The postoperative refractive outcome was taken as the spherical equivalent of the refraction at 3 months after surgery. The prediction error was taken as the absolute difference between the estimated and actual postoperative refraction. The data were analysed to assess the effects of age at the time of surgery, keratometry, and axial length on the accuracy of calculation of IOL power.

Results-For the overall group the mean and median prediction errors were $1.40 \mathrm{D}$ and $0.84 \mathrm{D}$ (SD 1.60). The mean and median prediction errors in eyes with axial lengths $\geqslant 20 \mathrm{~mm}$ were $1.07 \mathrm{D}$ and $0.71 \mathrm{D}$ (SD 0.98 ) and in eyes $<20 \mathrm{~mm}$ were $2.63 \mathrm{D}$ and 2.61 D (SD 2.65). The mean and median prediction errors in eyes in children aged $\geqslant 36$ months were $1.06 \mathrm{D}$ and $0.68 \mathrm{D}$ (SD 1.02) and in children aged <36 months was $2.56 \mathrm{D}$ and $2.29 \mathrm{D}$ (SD 2.50). The differences between the prediction errors for both axial length and age were statistically significant $(p<0.05)$.

Conclusions-For the overall group IOL power calculation is satisfactory. In eyes with axial lengths less than $20 \mathrm{~mm}$ and in children less than 36 months of age larger errors can arise. This study demonstrates the need for an IOL formula specifically designed for paediatric use.

(Br f Ophthalmol 2001;85:939-941)
\end{abstract}

Posterior chamber intraocular lens (IOL) implantation is gaining widespread use in the treatment of childhood cataract, as a consequence of advances in microsurgical techniques and IOL design. Recent reports have indicated that lens implantation in older children is a safe and effective treatment although not without some risk, including fibrinous uveitis, pupil capture, and capsular fibrosis. ${ }^{1-4}$ There is a growing trend towards IOL implantation surgery in infants, ${ }^{5}$ whose eyes are still undergoing rapid growth and refractive change. ${ }^{67}$ Many surgeons choose to aim for postoperative hypermetropia in young children and infants to allow for myopic shift as the eye grows. ${ }^{28-10}$ It is therefore fundamental that the calculation of IOL power should be as accurate as possible in giving a predictable postoperative refraction.

Many formulas have been devised, the first generation formulas being theoretically derived $^{1112}$ but proving to be inferior to the empirically derived formulas. ${ }^{13}$ Since then new generation formulas have come into existence which are modifications of previous theoretic and regression derived formulas including the SRK II and Holladay formulas. The SRK/T formula $^{14}$ was developed using theoretical formulas addressing the issues of postoperative anterior chamber depth, retinal thickness, and corneal refractive index optimised by regression analysis. Despite this refinement in IOL formulas, there is evidence that those in current use have lower predictive accuracy in eyes with very short axial lengths. ${ }^{15} 16$

\section{Methods}

All children (age $<16$ years) who underwent uncomplicated cataract surgery and IOL implantation between February 1994 and February 1998 were included in the study. We retrospectively reviewed the records of 40 children and data on 52 eyes are presented. All children had congenital or developmental cataract. No children in the group developed glaucoma preoperatively or postoperatively. Keratometry was performed using a Nidek hand held keratometer and axial length measurement using a Storz Compuscan. Measurements were taken on cooperative older children in clinic and on young children under general anaesthesia just before cataract surgery. Over the study period the SRK II and SRK T formulas were used, in conjunction with the IOL manufacturers' A constants, to calculate IOL power. All children $<12$ months of age at the time of surgery had IOL power calculated using the $\mathrm{SRK} / \mathrm{T}$ formula.

\section{SURGICAL TECHNIQUE}

Cataract surgery was performed through a tunnelled scleral incision. A keratome was used to enter the anterior chamber through clear cornea in a stepped fashion. Continuous circular capsulorhexsis or "can opener" capsulectomy (in surgery before 1995) was performed under viscoelastic. Lens material was removed using an automated irrigation/aspiration technique and the wound was enlarged to allow implantation of a rigid poly(methylmethacrylate) IOL into the capsular bag. Wound closure was performed using 10/0 Vicryl or 10/0 monofilament nylon sutures.

The power of the IOL implanted, the estimated postoperative refraction, and the age at the time of cataract surgery were recorded. 


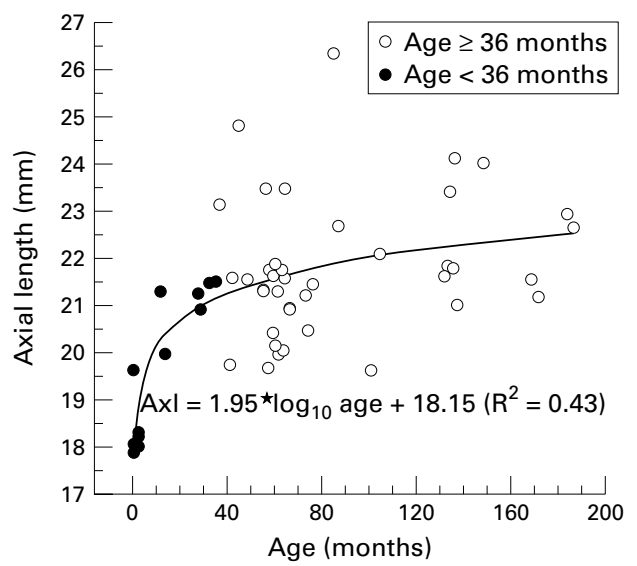

Figure 1 Axial length ( $\mathrm{mm}$ ) versus age at surgery (months).

The early postoperative refractive outcome was taken as the spherical equivalent of the refraction at 3 months after surgery. The absolute difference between estimated and actual postoperative refraction was calculated for all eyes and this was termed the prediction error. The data were analysed to assess the effects of axial length, keratometry, and age at the time of surgery on the accuracy of IOL power calculation. The differences between subgroups were analysed using the Mann-Whitney U test, with $\mathrm{p}$ $<0.05$ regarded as significant.

\section{Results}

Twelve children underwent bilateral IOL implantation and all children $<36$ months of age underwent unilateral IOL implantation surgery. Figure 1 shows age at time of surgery versus axial length.

Table 1 Prediction errors for the subgroup data

\begin{tabular}{llllll}
\hline & \multicolumn{5}{c}{ Prediction error (dioptres) } \\
\cline { 3 - 5 } & Number & Mean & Median & Range & \multirow{2}{*}{$S D$} \\
\hline Axial length $<20 \mathrm{~mm}$ & 11 & 2.63 & 2.61 & $0 .-9.19$ & 2.65 \\
Axial length $\geqslant 20 \mathrm{~mm}$ & 41 & 1.07 & 0.71 & $0.07-3.93$ & 0.98 \\
Corneal radii $<7.3 \mathrm{~mm}$ & 12 & 1.60 & 1.36 & $0.24-4.00$ & 1.3 \\
Corneal radii $\geqslant 7.3 \mathrm{~mm}$ & 40 & 1.32 & 0.76 & $0.07-9.19$ & 1.68 \\
Age at surgery $<36$ months & 12 & 2.56 & 2.29 & $0.24-9.19$ & 2.5 \\
Age at surgery $\geqslant 36$ months & 40 & 1.06 & 0.68 & $0.07-3.93$ & 1.02
\end{tabular}

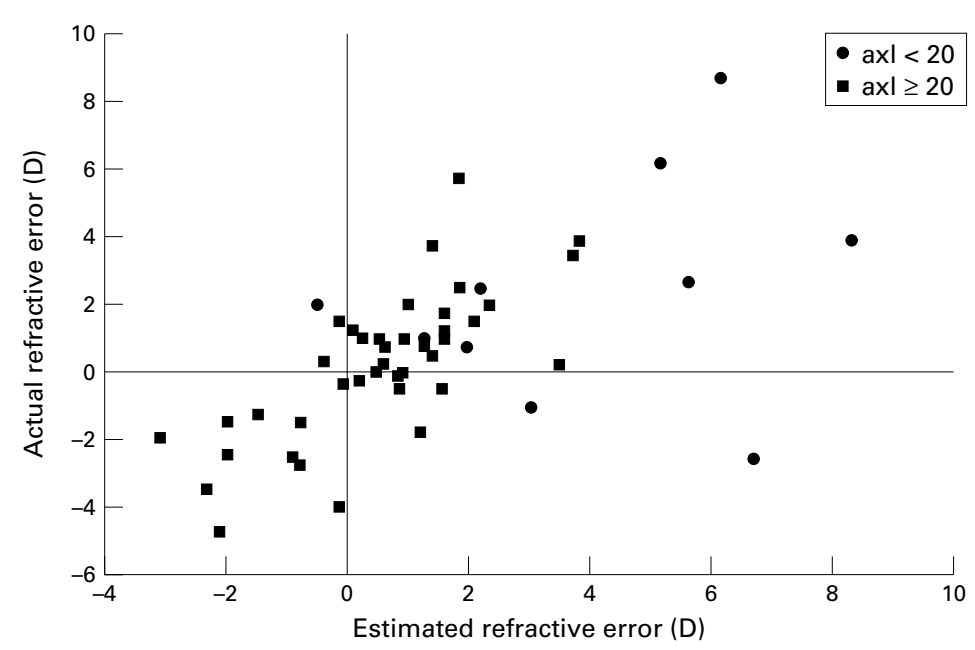

Figure 2 Estimated refractive error (D) versus actual refractive error for axial length (axl) $<20 \mathrm{~mm}$ and $\geqslant 20 \mathrm{~mm}$.
For the overall group of 52 eyes, axial length ranged from 17.9 to $26.36 \mathrm{~mm}$ (mean 21.34, median 21.41, SD 1.72), corneal radii from 6.58 to $8.43 \mathrm{~mm}$ (mean 7.57 , median 7.62 , SD 0.41 ), and age at the time of cataract surgery from 1 to 186 months (mean 70.48, median 60.5, SD 49.68).

Table 1 shows the prediction error data in the subgroups used for the statistical analysis. The data were subdivided for the eyes with axial lengths $<20 \mathrm{~mm}$ and $\geqslant 20 \mathrm{~mm}$, corneal radii $<7.3 \mathrm{~mm}$ and $\geqslant 7.3 \mathrm{~mm}$, and age at surgery $<36$ months and $\geqslant 36$ months.

For the overall group the prediction error ranged from 0.07 to $9.19 \mathrm{D}$ (mean 1.4 , median 0.84 , SD 1.6). Prediction error was significantly greater in eyes with axial lengths $<20$ $\mathrm{mm}(\mathrm{p}=0.04)$ and in eyes in children $<36$ months at the time of surgery $(p=0.03)$. There was a trend towards greater prediction error in eyes with corneal radii $<7.3 \mathrm{~mm}$, but this was not significant $(p=0.2)$.

Figure 2 shows a scatter plot of estimated refractive error versus actual refractive error for axial length $<20 \mathrm{~mm}$ and $\geqslant 20 \mathrm{~mm}$. This shows a tendency for the shorter eyes to have a lower actual than estimated refraction-for example, one of the eyes was estimated to be $+6.69 \mathrm{D}$ postoperatively but the actual refraction was $-2.50 \mathrm{D}$, creating a prediction error of $9.19 \mathrm{D}$. Other shorter eyes with estimated hypermetropic outcomes were found to be nearly emmetropic postoperatively.

\section{Discussion}

Several investigators have reported that formulas currently in use have lower predictive accuracy in eyes with shorter axial length. ${ }^{17}$ Our results show that for the overall group, the prediction error is satisfactory and is comparable with errors demonstrated in adult groups. ${ }^{16}$ In our study there was a trend towards a greater prediction error in eyes in children less than 36 months of age, in eyes shorter than $20 \mathrm{~mm}$, and in eyes with corneal radii less than 7.3 although for the latter, sample size is too small to reach significance.

Errors in calculating IOL power may arise in several ways:

\section{(1) INSTRUMENTATION ERROR}

Automated keratometry has been shown to be comparable to manual keratometry in adults. ${ }^{18}$ The steeper corneas of infants may result in inaccuracy, although the effect overall is likely to be small in the calculation of IOL power. Commercial biometers are optimally adapted for measurement of adult eyes and gates may not be set to receive echoes from shorter eyes (see Fig 2 for example). This was the case for Compuscan, which gave a minimum axial length of $17.8 \mathrm{~mm}$. It is therefore essential to ensure that instruments can be calibrated to accept shorter axial length readings. Most instruments use average sound velocity (1550 $\mathrm{m} / \mathrm{s}),{ }^{19}$ but in small eyes with congenital cataract the lens is a greater proportion of the total axial length and therefore the average velocity would be faster. Also in eyes with other 
abnormalities such as persistent hyperplastic primary vitreous the average sound velocity may be inappropriate.

(2) SURGICAL ERROR

Intraocular positioning of the IOL will affect the prediction error, with sulcus fixation producing a relative myopic shift from the estimated refraction. In our series four out of 52 IOLs had hybrid capsular bag/sulcus fixation and three of these eyes had a more myopic refractive error than was estimated. However, the sample size is too small to allow further analysis of this effect on prediction error.

(3) FORMULA ERROR

In our series SRK II and SRK/T formulas were used, although the results were recalculated for SRK/T for analysis of prediction error. It has been shown that the third generation theoretical formulas are more accurate for short eyes. This was attributed to their improved prediction of postoperative anterior chamber depth (ACD). However, the mean ACD in infant eyes is less than that of adult eyes ${ }^{20}$ and this may contribute to inaccuracy of IOL power calculation using current formulas. One strategy for improving prediction accuracy is to measure postoperative ACD and back calculate to modify the IOL formula as suggested by Holladay. ${ }^{21}$

In a recent study Inatomi ${ }^{22}$ showed that the SRK/T formula was more accurate than empirical formulas for calculating IOL power in eyes with microphthalmos (axial length $<19$ $\mathrm{mm}$ ) and they found increasing prediction error for the shorter eyes in their series.

(4) EYE GROWTH

Figure 1 showed that eyes that had surgery at age $<36$ months also tended to have shorter axial lengths. It is expected that there will be changes in the ocular dimensions in this group, especially when surgery is performed in the first few months of life. The resulting myopic shift ${ }^{9}{ }^{10}$ will lower the estimated refraction, and this should be borne in mind when comparing estimated and actual refractive outcomes.

In conclusion, the SRK/T formula for IOL power calculation gives satisfactory accuracy overall in predicting postoperative refractive error. However, in eyes with axial lengths shorter than $20 \mathrm{~mm}$ and in eyes in children less than 3 years of age unacceptably large prediction errors can arise. There is a trend towards greater prediction error in eyes with corneal radii $<7.3 \mathrm{~mm}$, although the sample size is too small for this to reach statistical significance. This study demonstrates the need to identify sources of calculation error, to study the rates of growth of implanted eyes, and to develop an IOL formula specifically designed for paediatric use.

The authors would like to thank Dr Neil Parry and Ms Sue Ritchie for their assistance in the preparation of this manuscript.

1 Plager DA, Lipsky SN, Snyder SK, et al. Capsular management and refractive error in pediatric intraocular lenses. Ophthalmology 1997;104:600-7.

2 Dahan E, Drusedau MUH. Choice of lens and dioptric power in pediatric pseudophakia. I Cataract Refract Surg 1997;23:618-23.

3 Ainsworth JR, Cohen S, Levin AV, et al. Pediatric cataract management with variations in surgical technique and aphakic optical correction. Ophthalmology 1997;104:1096101.

4 Zwaan J, Mullaney PB, Awad A, et al. Pediatric intraocular lens implantation. Ophthalmology 1998;105:112-19.

5 Awner S, Buckley EG, DeVaro JM, et al. Unilateral pseudophakia in children under 4 years. $\mathcal{F}$ Pediatr Ophthalmol Strabismus 1996;33:230-6.

6 Gordon RA, Donzis PB. Refractive development of the human eye. Arch Ophthalmol 1985;103:785-9.

7 Inagaki Y. The rapid change of corneal curvature in the neonatal period and infancy. Arch Ophthalmol 1986;104: neonatal pe 1026 .

8 McClatchey SK, Parks MM. Theoretic refractive changes after lens implantation in childhood. Ophthalmology 1997; 104: $1744-51$

9 Flitcroft DI, Knight-Nanan D, Bowell R, et al. Intraocular lenses in children: changes in axial length, corneal curvature and refraction. Br F Ophthalmol 1999;83:265-9.

10 Hutchinson AK, Drews-Botsch C, Lambert S. Myopic shift after intraocular lens implantation during childhood. Ophthalmology 1997;104:1752-7.

11 Collenbrander MC. Calculation of an iris clip lens for distance vision. Br f Ophthalmol 1973;57:735-40.

12 Binkhorst RD. The optical design of intraocular lens implants. Ophthalmic Surg 1975;6:17-31.

13 Sanders D, Retzlaff J, Kraff M, et al. Comparison accuracy of the Binkhorst, Colenbrander and SRK implant power prediction formulas. F Am Intraocul Implant Soc 1981;7: 337-40

14 Retzlaff J, Sanders D, Kraff M. Development of the SRK/T intraocular lens implant power calculation formula. $\mathcal{F}$ Cataract Refract Surg 1990;16:333-40.

15 Holladay JT, Gills JP, Leidlein J, et al. Achieving emmetropia in extremely short eyes with two piggyback posterior chamber intraocular lenses. Ophthalmology 1996;103:1118-23.

16 Andreo LK, Wilson ME, Saunders RA. Predictive value of regression and theoretical IOL formulas in pediatric intraocular lens implantation. F Pediatr Ophthalmol Strabismus 1997;34:240-3.

17 Olsen T, Thim K, Corydon L. Accuracy of the newer generation intraocular lens power calculation formulas in generation intraocular lens power calculation formulas in 93.

18 Manning CA, Kloess PM. Comparison of portable automated keratometry and manual keratometry for IOL calculation. F Cataract Refract Surg 1997;23:1213-16.

19 Jansson F, Koch E. Determination of the velocity of ultrasound in the human lens and vitreous. Acta Ophthalmol 1962;40:420-33

20 Larsen JS. The sagittal growth of the eye. 1. Ultrasonic measurement of the depth of the anterior chamber from birth to puberty. Acta Ophthalmol 1971;49:239-62.

21 Holladay JT. Standardizing constants for ultrasonic biometry, keratometry and intraocular lens power calculations. $\mathcal{F}$ Cataract Refract Surg 1997;23:1356-70.

22 Inatomi $\mathrm{M}$, Ishii $\mathrm{K}$, Koide $\mathrm{R}$ et al. Intraocular lens power calculation for microphthalmos. F Cataract Refract Surg 1997;23:1208-12. 